\section{Span Quaternary fields}

Pedology, Weathering, and Geomorphological Research. By Peter W. Birkeland. Pp. xiii +285 . (Oxford University Press: London and New York, October 1974.) £6.25.

FEw books set out to bridge the considerable gaps between the various disciplines in Quaternary studies. This may be a reflection of the range and diversity of material, and consequently of the specialisms demanded of the author. It must, therefore, have required a commendable boldness to embark on a work as complicated as that suggested by the title of Professor Birkeland's new book.

The text spans the interesting and fruitful field between pedology and other Quaternary studies, but each reader will probably have a different view of the likely contents, and may therefore be frustrated by what is actually covered. The title does not represent the emphasis accurately. 'The major stress lies on the factors of soil formation (chapters 6-11), but there are introductory chapters on soil profiles, properties and classification (chapters 1 and 2), a section on weathering and soil forming processes (chapters 3-5), and a final single chapter on the use of soils in Quaternary studies. Pedologists will be familiar with most of these topics, and anyone interested in weathering and soil processes will find the material rather brief but succinct.

Geomorphologists may be thwarted in their search for appropriate material as there is no chapter specifically upon geomorphological work. Topics of recent geomorphic interest, such as quantitative research into processes, slope analysis and chronology are barely mentioned. The level is more appropriate to senior undergraduate and first year postgraduate students in British universities.

The text is geared to the American market. Nearly all the examples are taken deliberately from North America, and it is a matter of some regret that there are relatively few publications discussed, even in English, from outside that area. The depth of recommended reading varies from 73 references for one chapter down to 11 for another.

The lack of a review of biological factors leads to some imbalance; neither the chapter on vegetation and soil relationships (which fails to consider either fauna or microorganisms), ror the final chapter give adequate consideration to the biotic influences on soil development.

Despite these general criticisms and a number of minor quibbles, this book makes worthwhile reading on several counts. It does present a summary of recent (particularly American) research and it does bring together the research of diverse but related fields. Amplification of the topics presented, and a more extensive review of allied geomorphological research would add to the value of any future editions.

Peter A. Furley

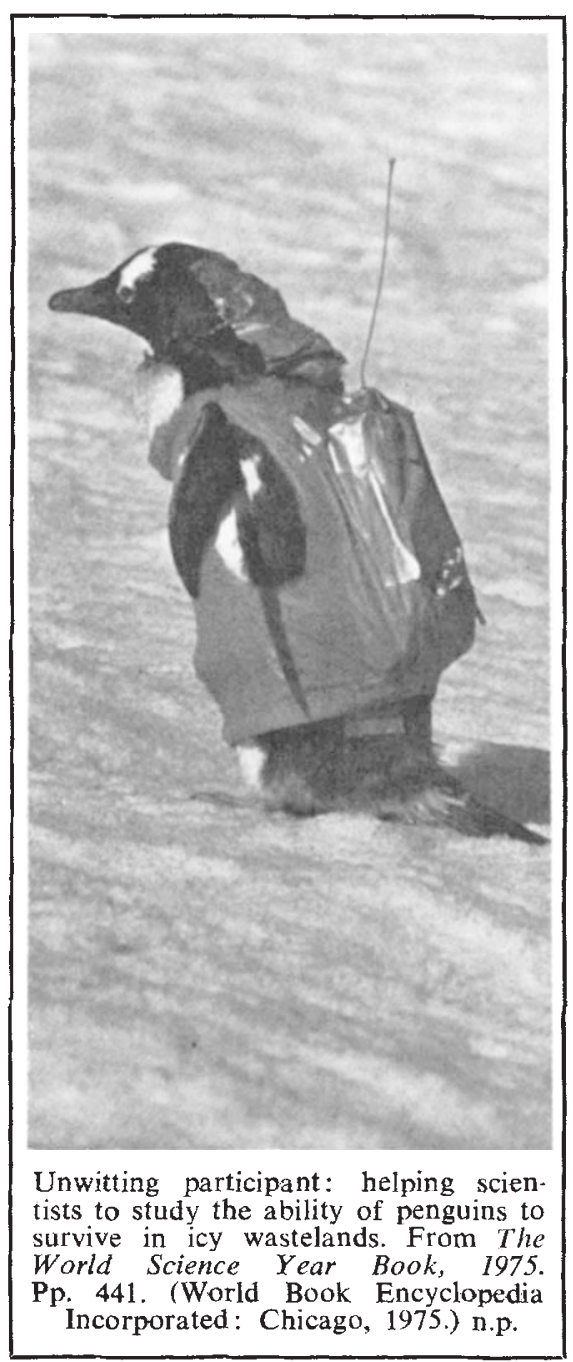

\section{Bubble devices}

Magnetic Bubbles. By T. H. O'Dell. Pp. $\mathrm{x}+159$. (Macmillan: London and Basingstoke, January 1975.) $£ 8.50$.

Magnetic Bubbles will prove invaluable in a variety of roles; it will, for example, provide an excellent introduction and foundation course for device engineers and materials scientists entering the new field of magnetic bubble devices. In spite of the rapidly growing bibliography on magnetic bubbles the extensive references provided by the author will considerably aid established workers in this field to recover previously published information. The author has concentrated on the underlying physics of both the operation of devices and the materials used. The treatment has emphasised, where appropriate, the mathematical aspects of the subject, and in most cases it has been done in such a way as to inspire confidence, maintaining a balance between the basic philosophy of bubble devices and a rigorous mathematical treatment.

Consider a thin layer of magnetic material which, because of its structure, can easily be magnetised to saturation only in directions normal to itself. A magnetic bubble is a small and mobile cylindrical region, extending through the layer, which is magnetised in a sense opposing the remainder of the layer. Bubbles are used for data processing and storage, each one representing a ' 1 ' digit. A bubble device consists simply of an assembly of a number of integrated circuits each of which carries magnetically activated tracks, that is, shift registers, along which are driven patterns of bubbles and gaps representing binary data.

Chapters on magnetostatics and bubble dynamics are particularly well presented, bringing together a well balanced explanation incorporating a number of basic concepts which previously have been scattered among a handful of technical articles. These chapters would be valuable in aiding a first degree course in magnetics.

Two chapters deal with areas close to device design and technology. One provides a review of the range of magnetic materials which have been studied as candidates for bubble devices and explains the important characteristics which ensure adequate bubble stability. Although sufficient detail is given on garnet layers-which are prepared by liquid phase epitaxy - to indicate that they represent a viable device basis the author has omitted details on the epitaxy process itself. That is a pity because were such detail included it would be clear that the models used to represent the layers are idealised in that available layers actually possess nonuniformities in composition throughout their thickness. The other sections dealing with devices provide an excellent treatment of bubble motion in the potential wells produced by the shift register elements. The author's approach is, I believe, unique and is certainly likely to inspire the device engineer.

This review would be incomplete were I not to report the one error I found. Although nothing which follows it is invalidated, the approximation used by the author in Chapter 3 for the exchange constant may mislead readers to assume that the exchange constant i.s virtually independent of temperature. Actually, it decreases monotonically with increasing temperature, reaching zero at the Curie temperature.

Anthony Marsh 